\title{
Analysis of radiation-induced genome alterations in Vigna unguiculata
}

This article was published in the following Dove Press journal:

Research and Reports in Biology

3I August 201 I

Number of times this article has been viewed

\section{Christell van der Vyver' \\ B Juan Vorster ${ }^{2}$ \\ Karl J Kunert ${ }^{3}$ \\ Christopher A Cullis ${ }^{4}$}

'Institute for Plant Biotechnology, Department of Genetics, University of Stellenbosch, Stellenbosch, South Africa; ${ }^{2}$ Department of Plant Production and Soil Science, and ${ }^{3}$ Department of Plant Science, Forestry and Agricultural Biotechnology Institute, University of Pretoria, Pretoria, South Africa; ${ }^{4}$ Case Western Reserve University, Department of Biology, Cleveland, $\mathrm{OH}$, USA
Correspondence: Christell van derVyver Institute for Plant Biotechnology, Department of Genetics, University of Stellenbosch, Stellenbosch 7602, South Africa

Tel +27218083834

Fax +27218083835

Email cvdv@sun.ac.za
Abstract: Seeds from an inbred Vigna unguiculata (cowpea) cultivar were gamma-irradiated with a dose of $180 \mathrm{~Gy}$ in order to identify and characterize possible mutations. Three techniques, ie, random amplified polymorphic DNA, microsatellites, and representational difference analysis, were used to characterize possible DNA variation among the mutants and nonirradiated control plants both immediately after irradiation and in subsequent generations. A large portion of putative radiation-induced genome changes had significant similarities to chloroplast sequences. The frequency of mutation at three of these isolated polymorphic regions with chloroplast similarity was further determined by polymerase chain reaction screening using a large number of individual parental, M1, and M2 plants. Analysis of these sequences indicated that the rate at which various regions of the genome is mutated in irradiation experiments differs significantly and also that mutations have variable "repair" rates. Furthermore, regions of the nuclear DNA derived from the chloroplast genome are highly susceptible to modification by radiation treatment. Overall, data have provided detailed information on the effects of gamma irradiation on the cowpea genome and about the ability of the plant to repair these genome changes in subsequent plant generations.

Keywords: mutation breeding, gamma radiation, genetic mutations, cowpea, representational difference analysis

\section{Introduction}

Cowpea (Vigna unguiculata) is one of the most important tropical multipurpose legumes used as a protein source for human nutrition in Africa. ${ }^{1}$ Ionizing radiation has been used in cowpea mutation breeding programs to generate genetic variation for crop improvement by inducing heritable chromosomal changes at specific loci. ${ }^{2-5}$ Mutations known to occur include altered, missed, or mismatched nucleotide bases, DNA sequence insertions and deletions, linking of pyrimidines, and double-stranded DNA breaks as well as intrastrand and interstrand crosslinking. ${ }^{2,3,5-8}$

The level of induced mutations is a function of the irradiation dose, and a study in Arabidopsis suggests a linear relationship between dose and effect. ${ }^{9-11}$ Although the location of these changes cannot be directed, distribution of the mutations in the genome does not appear to be random, with mutation frequencies varying significantly across the genome so that certain positions (hotspots) can be identified. ${ }^{12}$ An irradiation experiment on transgenic Arabidopsis seeds showed no significant correlation between detected mutation rate within a specific transgene and the irradiation dose. However, a significant difference in mutation rate was found among 15 different loci of Arabidopsis exposed to electromagnetic spectra, X-rays, and fast neutrons. ${ }^{9,13}$ Generally, mutations induced by irradiation are produced in close proximity to each 
other, usually within one-two helical turns of the DNA molecule leading to clustered DNA damage. ${ }^{14,15}$ Detailed knowledge about these lesions is still rather limited. The genes in which mutations have been identified have mainly been involved in plant growth regulation and senescence processes or those influencing plant morphology due to their ease of detection. ${ }^{5,16-18}$

Given that little information is currently available regarding the genome-wide effect of ionizing radiation, this study was designed to perform a genome-wide analysis of the variation induced by ionizing radiation in cowpea. A specific objective of this study was to isolate and characterize such possible mutational hotspots affected by radiation treatment. Representational difference analysis (RDA) was used to identify and isolate mutated regions in the cowpea genome following irradiation. ${ }^{19-21}$ In conjunction with RDA, the mutated genomes were further characterized using random amplified polymorphic DNAs (RAPDs), which have been successfully used in plants to detect "DNA effects" induced by radiation. ${ }^{22}$ RAPDs have been further used to show that DNA damage after gamma irradiation of Vigna radiate is dose-dependent. ${ }^{10,23,24}$ Simple sequence repeat (SSR) markers were also used to screen the different mutant lines for the presence of induced polymorphisms and exclude outcrossing as a possible source of the genomic variation. Here we report about the measurement of the initial damage to the cowpea genome as a result of ionizing radiation by screening M1 plants, the genomic regions affected, and the persistence of the mutations in these highly variable regions through subsequent generations.

\section{Materials and methods}

\section{Mutagenesis and plant material}

Dry cowpea seeds (IT93K129-4) obtained from the International Institute of Tropical Agriculture were exposed to a cobalt-60 gamma source (International Atomic Energy Agency, Siebersdorf, Austria) for irradiation at $25^{\circ} \mathrm{C}$ using an exposure dose of 180 Gy. Irradiated seeds (17,700 seeds in total) were sown and M2 seeds (8034 seeds in total) were harvested. Seeds from different M2 mutated lines were planted, harvested, and replanted in four consecutive growth seasons to obtain the M3, M4, M5, and M6 generations (mutant collection were done by Spreeth et al at the Agricultural Research Council, South Africa).

The plant material used in this study consisted of two groups. The first group included seeds of the control parent line, IT93K129-4, and seeds of eight irradiation-generated mutant lines (lines 217, 164, BO, BQ, 26, MA1, MA2, and I) for which the M2-M6 generations (mutant generations 2-6) were available. The second group included seeds of the control parent line, IT93K129-4, and irradiated mutant individuals from the M1 generation.

\section{DNA isolation}

Total DNA was isolated using the Qiagen DNEasy kit (Whitehead Scientific, Cape Town, South Africa) from control and mutant cowpea plant leaves or from the embryonic axis of irradiated M1 seeds that did not germinate. In this way, all 100 irradiated M1 seeds were sampled to determine DNA mutation events.

\section{Representational difference analysis}

The general outline for the RDA procedure was followed previously described. ${ }^{25,26} \mathrm{~A}$ genome representation was prepared by digestion of $2 \mu \mathrm{g}$ of each of the DNAs separately with the restriction enzymes, $M b o \mathrm{I}, M s p \mathrm{I}$, or Csp6I (Fermentas, Inqaba Biotech, Pretoria, South Africa). The digests were then ligated to the first set of the appropriate adaptors. The ligation products were amplified by the polymerase chain reaction (PCR) and the amplification products (amplicons) were digested with the appropriate restriction enzyme to remove the adaptors. Tester DNA was prepared by adding a second adaptor pair to the ends of the first round amplicons. The driver DNA consisted of the remainder of the amplicons following adaptor removal. After each round of subtraction, the adaptor sequences attached to the tester DNA were replaced by a different adaptor sequence. Table 1 summarizes the combinations of driver and tester DNAs used for the RDA subtractions.

The first round of subtractive hybridization was done at a $50: 1$ driver/tester ratio, the second round at a ratio of 500:1 (200:1 for Csp6I), and a third round at a ratio of 5000:1 (2000:1 for Csp6I). The final subtraction products were filled in, blunt end-ligated into the pMOSBlue cloning vector (Amersham Pharmacia Biotech, Little Chalfont, UK) and transferred into Escherichia coli-competent cells (Amersham Pharmacia Biotech, UK). Plasmid-containing colonies carrying an insert were randomly selected and sequenced. Primers were designed for each of these difference products. The primer pairs were used in standard PCR reactions using genomic cowpea DNA as a template for genome screening. All PCRs were repeated three times, and the same template DNA preparation was used for all primer sets listed to ensure consistent results. PCR reactions were carried out in $50 \mu \mathrm{L}$ volumes containing $40 \mathrm{ng}$ of total genomic DNA, $15 \mathrm{ng}$ of primer, $100 \mathrm{mM}$ of each dNTP, $10 \mathrm{mM}$ Tris- $\mathrm{HCl}, \mathrm{pH} 8.3,2 \mathrm{mM} \mathrm{MgCl}_{2}$ and 0.5 units of Taq polymerase (TaKaRa, Separations, Johannesburg, South Africa). Amplification was performed using a Perkin Elmer GeneAmp PCR system 9600. The following standard 
Table I RDA subtraction carried out with different control and mutant cowpea lines

\begin{tabular}{|c|c|c|c|}
\hline & RDA experiment & Driver & Tester \\
\hline \multirow[t]{3}{*}{ Mspl } & $\mathrm{I}(\mathrm{EI})$ & Control line (IT93K I 29-4) & $\begin{array}{l}\text { Mutant lines = MAI-M3, MA2-M3, 2I7-M4, } \\
\text { I64-M4, BQ-M3, 26-M5 and I-M3 }\end{array}$ \\
\hline & $2(\mathrm{E} 2)$ & Mutant line MAI-M3 & Control line (IT93K I29-4) \\
\hline & $3(\mathrm{E} 3)$ & Mutant line 2I7-M4 & Control line (IT93K I29-4) \\
\hline \multirow[t]{4}{*}{ Mbol } & $5(\mathrm{E} 5)$ & Control line (IT93K I 29-4) & Mutant lines MAI-M3 and 2I7-M4 \\
\hline & $6(\mathrm{E} 6)$ & Mutant line MAI-M3 & Control line (IT93K I29-4) \\
\hline & 7 (E7) & Mutant line 217-M4 & Control line (IT93K I29-4) \\
\hline & 8 (E8) & Control line (IT93K I 29-4) & Mutant line 217,10 individuals, M2 \\
\hline \multirow[t]{2}{*}{ Csp6I } & 9 (E9) & IT96D-602 & Mutant line $164-M 2$ \\
\hline & II (EII) & Mutant line $164-M 2$ & Control line IT93K I29-4 \\
\hline
\end{tabular}

Notes: Nine RDA experiments were done with three different restriction enzymes, namely Mspl, Mbol, and Csp6l. Equal concentrations of genomic DNA were mixed for multiple sample tester DNAs.

Abbreviation: RDA, representational difference analysis.

amplification program was used: $94^{\circ} \mathrm{C}$ for five minutes $\times$ one cycle; $94^{\circ} \mathrm{C}$ for one minute, $55^{\circ} \mathrm{C}$ or $60^{\circ} \mathrm{C}$ for one minute depending on the primer pair, $72^{\circ} \mathrm{C}$ for one minute $\times 35$ cycles; $72^{\circ} \mathrm{C}$ for five minutes $\times$ one cycle followed with an optional soak period at $4^{\circ} \mathrm{C}$. The PCR products were separated on a $1.5 \%$ agarose gel, stained with ethidium bromide, and visualized under ultraviolet light.

\section{RAPD analysis}

RAPD primers OPA06, OPB01, OPB07, OPB11, OPC08, OPC10, OPC11, OPC12, OPP14, OPK04, OPAC17, and OPAC19 (Operon Technologies, Qiagen, Whitehead Scientific, Cape Town, South Africa) were tested for detection of cowpea polymorphisms (Table 2), using similar PCR conditions as above with an annealing temperature of $42^{\circ} \mathrm{C}$.

\section{SSR analysis}

A modified FIASCO SSR isolation protocol followed using primers $\mathrm{J}-C s p-12$ and $\mathrm{J}-C s p-24$ and Csp6I digested the genomic IT93K129-4 control cowpea DNA. ${ }^{27}$ SSR-enriched fractions were cloned after PCR using the TOPO-TA cloning kit (Invitrogen, Celtic Molecular Diagnostic, Johannesburg, South Africa). Plasmids from selected clones were isolated and sequenced. The sequences flanking the repeats were used to design specific primers for PCR amplification across the SSRs. The SSRs were analyzed in 100 M1 cowpea individuals

Table 2 Nucleotide sequences of primers used in the RAPD and SSR assays

\begin{tabular}{|c|c|c|c|}
\hline SSR & Sequence & RAPD & Sequence \\
\hline CP-SSR-I (L) & 5'-AGTCCCATGCCСTCTCTTCT-3' & OPBII & 5'-GTAGACCCGT-3' \\
\hline CP-SSR-I (R) & 5'-GCCTCTCACCGATAGCACTC-3' & OPB07 & 5'-GGTGACGCAG-3' \\
\hline CP-SSR-2(L) & 5'-TTGCTTCATCGCATTTTCAA-3' & OPACI9 & 5'-AGTCCGCCTG-3' \\
\hline CP-SSR-2(R) & 5' GCTGAACCTGGCACAGTAGA-3' & OPK4 & $5^{\prime}-$ CCGCCCAAAC- $3^{\prime}$ \\
\hline CP-SSR-3(L) & 5'-CGCATGAAAGCTTGTTGGTA-3' & OPCII & 5'-AAAGCTGCGG-3' \\
\hline CP-SSR-3(R) & 5'-GGCTGAAGGAGAAAATACGAAA-3' & OPA06 & 5'-GGTCCCTGAC-3' \\
\hline CP-VM5(L) & 5'-AGCGACGGCAACAACGAT-3' & OPBOI & 5'-GTTTCGCTCC-3' \\
\hline CP-VM5(R) & 5'-TTCCCTGCAACAAAAATACA-3' & OPACI7 & 5'-CCTGGAGCTT-3' \\
\hline CP-VMI3(L) & 5'-CACCCGTGATTGCTTGTT G-3' & OPPI 4 & $5^{\prime}-$ CCAGCCGAAC- $3^{\prime}$ \\
\hline CP-VMI3(R) & 5'-GTCCССТСССТСССАСТG-3' & $\mathrm{OPCl} 2$ & 5'-TGTCATCCCC-3' \\
\hline CP-VM3I (L) & 5'-CGCTCTTCGTTGATGGTTATG-3' & OPCIO & 5'-TGTCTGGGTG-3' \\
\hline CP-VM3I(R) & 5'-GTGTTCTAGAGGGTGTGATGGTA-3' & OPC08 & 5'-TGGACCGGTG-3' \\
\hline CP-VM35(L) & 5'-GGTCAATAGAATAATGGAAAGTGT3' & & \\
\hline CP-VM35(R) & 5'-ATGGCTGAAATAGGTGTCTGA - $3^{\prime}$ & & \\
\hline CP-VM36(L) & 5'-ACTTTCTGTTTTACTCGACAACTC-3' & & \\
\hline CP-VM36(R) & 5'-GTCGCTGGGGGTGGCTTATT-3' & & \\
\hline CP-VM39(L) & 5'-GATGGTTGTAATGGGAGAGTC-3' & & \\
\hline CP-VM39(R) & 5'-AAAAGGATGAAATTAGGAGAGCA-3' & & \\
\hline CP-VM7I (L) & 5'-TCGTGGCAGAGAATCAAAGACAC-3' & & \\
\hline CP-VM7I(R) & 5'-TGGGTGGAGGCAAAAACAAAA-3' & & \\
\hline
\end{tabular}

Abbreviations: RAPD, random amplified polymorphic DNAs; SSR, simple sequence repeat. 
derived from the same irradiation event using three primer sets developed in this study (CP-SSR 1-3) as well as seven primer sets obtained from a previous study ${ }^{28}$ (VM5, VM13, VM31, VM35, VM36, VM39, and VM71, see Table 2) using PCR conditions similar to those described above.

\section{Results}

\section{Isolation of RDA subtraction products from cowpea}

Twenty-two subtraction products were characterized. Nine had similarity to cowpea chloroplast-like sequences (http:// cowpeagenomics.med.virginia.edu/CGKB/) (Table 3); one had similarity to satellite DNA (X87233.1) and a resistance gene analog (AF534321.1); one had similarity to a legume 26S ribosomal RNA gene (AY935814.1); one had similarity to repetitive DNA (EGU271985); and one showed some similarity to a putative retrotransposon polyprotein (AAV88076.1). The remaining subtraction products showed no significant similarity to any reported database sequences. Primers were designed for all these subtraction products, and genomic DNA from various control and mutated individuals were used as templates for genome screening.

\section{Nuclear plastidic insertions}

\section{Subtraction product E2/D}

Subtraction product E2/D was a sequence isolated from a single mutant plant of the M3 generation of line MA1 subtracted with the nonirradiated parent line DNA. The subtraction product showed significant similarity to a V. unguiculata chloroplast fragment (AF141146). Primers E2LD and E2RD were designed (Table 4) based on the known Vigna sequence flanking subtraction product E2/D. In order to distinguish possible nuclear copies of chloroplast-related sequences from those in the chloroplast (the latter not containing 5-methyl cytosine ${ }^{29}$ ), the genomic template DNA from both control and mutant individuals was first digested with the methylation-sensitive restriction enzyme $M s p I$ which was followed by PCR using primers E2LD and E2RD. PCR products were amplified from tested control and mutant cowpea plants, with possibly varying numbers of copies due to difference in amplicon intensity on the gel, with the exception of one 217 M2 individual plant which failed to amplify a fragment (Figure 1, lane 9). Sequence analysis revealed a 13 bp deletion in one $217 \mathrm{M} 2$ mutant plant and a single point mutation ( $\mathrm{T}$ deletion) in a $217 \mathrm{M} 4$ mutant cowpea plant.

Table 3 Summary of significant sequence similarities of isolated RDA subtraction products to chloroplast-like sequences (including mutations identified in the isolated RDA subtraction products)

\begin{tabular}{|c|c|c|c|c|}
\hline $\begin{array}{l}\text { Difference } \\
\text { product }\end{array}$ & Size & NCBI similarity & GCKB similarity & Mutations \\
\hline$E 2 / D$ & 107 bp & $\begin{array}{l}\text { AFI } 4 \text { I I } 46 \\
\text { Vigna unguiculata Id: 107//07; 3e-48 }\end{array}$ & $\begin{array}{l}\text { gnl/vuna/3498I } 976 \\
\text { Id: } 107 / 107 ; 6.8 \mathrm{e}-55\end{array}$ & Methylation changes \\
\hline$E 3 / 3$ & 389 bp & $\begin{array}{l}\text { EUI } 96765 . \text { I Phaseolus vulgaris chloroplast } \\
\text { Id:387/389; e = } 0\end{array}$ & $\begin{array}{l}\text { gnl/vuna/34981 } 976 \\
\text { Id:388/389; e = } 0\end{array}$ & $\mathrm{C}$ to $\mathrm{T}$ point mutation \\
\hline $\mathrm{E} 5 / 8$ & 162 bp & $\begin{array}{l}\text { EUI } 96765 . \text { I Phaseolus vulgaris chloroplast } \\
\text { Id: 148/I5I; 2e-67 } \\
\text { AP002983.I Lotus corniculatus }\end{array}$ & $\begin{array}{l}\text { gnl/vuna/3498I976 } \\
\text { Id:I49/I5I; 3.6e-76 }\end{array}$ & $\begin{array}{l}\text { Two } T \text { to } C \text { point mutations, } \\
\mathrm{I} 2 \text { bp mismatch at end }\end{array}$ \\
\hline $\mathrm{EI} / 3 \mathrm{C}$ & 178 bp & $\begin{array}{l}\text { EUI 96765.I Phaseolus vulgaris chloroplast } \\
\text { Id: 175/I78; 2e-82 }\end{array}$ & $\begin{array}{l}\text { gnl/vuna/3498I } 976 \\
\text { Id: I 76/I78; 3.I e-92 }\end{array}$ & $\begin{array}{l}\mathrm{G} \text { to } \mathrm{A} \text { and } \mathrm{T} \text { to } \mathrm{C} \text { point } \\
\text { mutations }\end{array}$ \\
\hline $\mathrm{EI} / 3 \mathrm{~A}$ & $89 \mathrm{bp}$ & $\begin{array}{l}\text { GQ893027.I } \\
\text { Vigna radiata chloroplast DNA } \\
\text { Id:86/92; } 9 \text { e- } 29\end{array}$ & $\begin{array}{l}\text { gnl/vuna/3498I } 976 \\
\text { Id:89/89; 3e-44 }\end{array}$ & Plastid insert \\
\hline $\mathrm{El} / 3 \mathrm{~B}$ & $201 \mathrm{bp}$ & $\begin{array}{l}\text { GQ893027.I } \\
\text { Vigna radiata chloroplast DNA } \\
\text { Id:196/203; } 2 \mathrm{e}-88\end{array}$ & $\begin{array}{l}\text { gnl/vuna/3498I } 976 \\
\text { Id:196/I97; I.6e-103 }\end{array}$ & Plastid insert, TGGG \\
\hline$E 2 / A$ & $230 \mathrm{bp}$ & $\begin{array}{l}\text { GQ893027.I } \\
\text { Vigna radiata chloroplast DNA } \\
\text { Id: } 226 / 233 ; 6 \mathrm{e}-105\end{array}$ & $\begin{array}{l}\text { gnl/vuna/3498। } 976 \\
\text { Id: } 229 / 230 ; 1.5 \mathrm{e}-125\end{array}$ & $\begin{array}{l}\text { Plastid insert, } A \text { to } G \text { point } \\
\text { mutation }\end{array}$ \\
\hline$E 2 / B$ & 107 bp & $\begin{array}{l}\text { GQ893027.I } \\
\text { Vigna radiata chloroplast DNA } \\
\text { Id: } 103 / 110 ; 5 \mathrm{e}-37\end{array}$ & $\begin{array}{l}\text { gnl/vuna/3498I } 976 \\
\text { Id:I06/I07; I.6e-52 }\end{array}$ & $\begin{array}{l}\text { Plastid insert } G \text { to } A \text { point } \\
\text { mutation }\end{array}$ \\
\hline $\mathrm{E} 2 / \mathrm{C}$ & 107 bp & $\begin{array}{l}\text { GQ893027.I } \\
\text { Vigna radiata chloroplast DNA } \\
\text { Id: I04/I I0; I e-38 }\end{array}$ & $\begin{array}{l}\text { gnl/vuna/3498I976 } \\
\text { Id:I07/I07; 6.8e-55 }\end{array}$ & Plastid insert \\
\hline
\end{tabular}

Abbreviations: CGKB, cowpea gene space sequence knowledge base; NCBI, National Center for Biotechnology Information. 
Table 4 Nucleotide sequences of primers designed for the RDA subtraction products E2/D and E5/8

\begin{tabular}{lll}
\hline $\begin{array}{l}\text { RDA } \\
\text { product }\end{array}$ & Primer & Sequence \\
\hline E2/D & E2LD & 5'-GAGGACGGGTTTTT GGAGTT-3' \\
& E2RD & 5'- CGTGTACACAGGTGGTGCAT-3' \\
E5/8 & E5L8 & 5' ATCGTGTATTTCATTCTAGTTCGATG -3' \\
& E5R8 & 5' GATCCACGAACGGATCCGAAATCT-3' \\
& E5L8out & 5'-T CCCGATTCATGGATCTCTC-3' \\
& E5L8out & 5'-CTTCCAAATTA TTCCCGCAA-3' \\
\hline
\end{tabular}

Abbreviation: RDA, representational difference analysis.

\section{Subtraction product $E 5 / 8$}

Subtraction product E5/8 was isolated using the restriction enzyme $\mathrm{MboI}$ from the pooled DNA of two mutant plants of lines MA1 and 217 as the tester and the nonirradiated parental line as the driver (Table 1). The product had significant similarity with chloroplast DNAs of Lotus corniculatus var japonicus chloroplast (AP002983.1) and V. unguigulata (gn/ vuna/34981976). The last 12 bp of E5/8 show no overlap with any known database sequences. Therefore, it is possible that this subtraction product is a nuclear-derived fragment where part of the extant chloroplast DNA is present in the nuclear genome. ${ }^{30}$ Primers that amplify within and across this region were designed (Table 4). A primer pair E5L8 and E5R8 was designed to allow E5L8 binding within the unknown 12 bp region of E5/8. The E5/8 region was found to be present in all nonirradiated control individuals but only in $70 \%$ of the mutant individuals (Figure 2). Primer pair E5L8out and E5R8out was designed using a Vigna chloroplast sequence that flanked subtraction product E5/8. This primer set resulted in an amplification product in all the nonirradiated control individuals but in only $26 \%$ of the mutant individuals (Figure 3). Ten bases are different

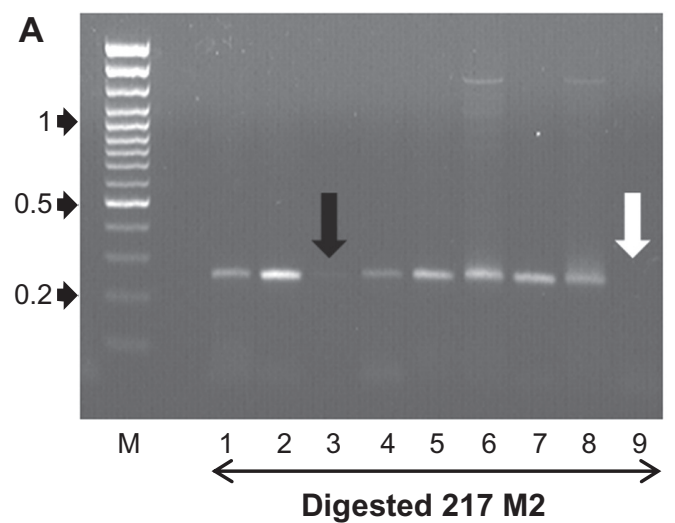

between the difference product and the Vigna chloroplast sequence.

E5L8out and E5R8out were used to amplify the DNA isolated from $100 \mathrm{M} 1$ individuals to investigate the frequency of irradiation-linked mutations in this chloroplast-like region. Lack of a PCR amplification product was interpreted as the parent being homozygous for lack of the target PCR fragment or the primer sequences. Presence of a PCR amplification product was interpreted as the parent being either homozygous or heterozygous for the presence of the PCR target or the primer sequences. Amplification of both of these two regions was achieved with all nonirradiated control individuals tested. However, only $68 \%$ and $91 \%$ of the M1 mutant individuals showed amplification with primers sets E5/8 and E5/8out, respectively, indicating that DNA damage in this region was present in at least 32\% (E5/8) and 9\% (E5/8out) of the M1 irradiated individuals, respectively. Because the data do not reflect the number of heterozygous individuals, the rate of change at this locus is likely to be much higher than these $32 \%$ and $9 \%$ values.

An equal number of $\mathrm{M} 1$ individuals, with or without DNA damage in region $\mathrm{E} 5 / 8$, were selfed. The $\mathrm{M} 2$ seeds were planted and genomic DNA extracted for further PCR screening. Of the M2 individuals screened (M1 DNA homozygous for damage group), $37.5 \%$ still showed no amplification of the E5/8 fragment, while in $62.5 \%$ of the individuals (from which this fragment was absent in the M1 line) this fragment could be amplified. The screened M2 individuals (M1 no apparent mutation group) segregated into $25 \%$ showing no amplification versus $75 \%$ showing amplification of $\mathrm{E} 5 / 8$. This is consistent with the M1 generation being heterozygous for this region (mutated/nonmutated) because the expected 3:1 segregation for a dominant marker was observed. Therefore, the data indicate

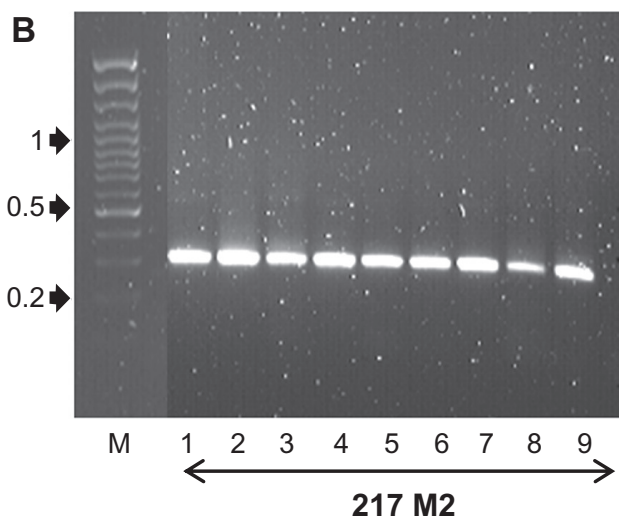

Figure I Genomic cowpea DNA used as template in a PCR reaction with primer set E2LD and E2RD. (A) Lanes I-9 represent Mspl digested and amplified DNA from nine individual plants of mutant cowpea line 217, M2 generation. (B) Lanes I-9 represent corresponding undigested and amplified DNA from individuals I-9 of mutant line 217 , M2 generation. Lane M represents a molecular DNA marker in kbp. Black arrow indicates a weak PCR product and white arrow the absence of a PCR product. Abbreviation: PCR, polymerase chain reaction. 

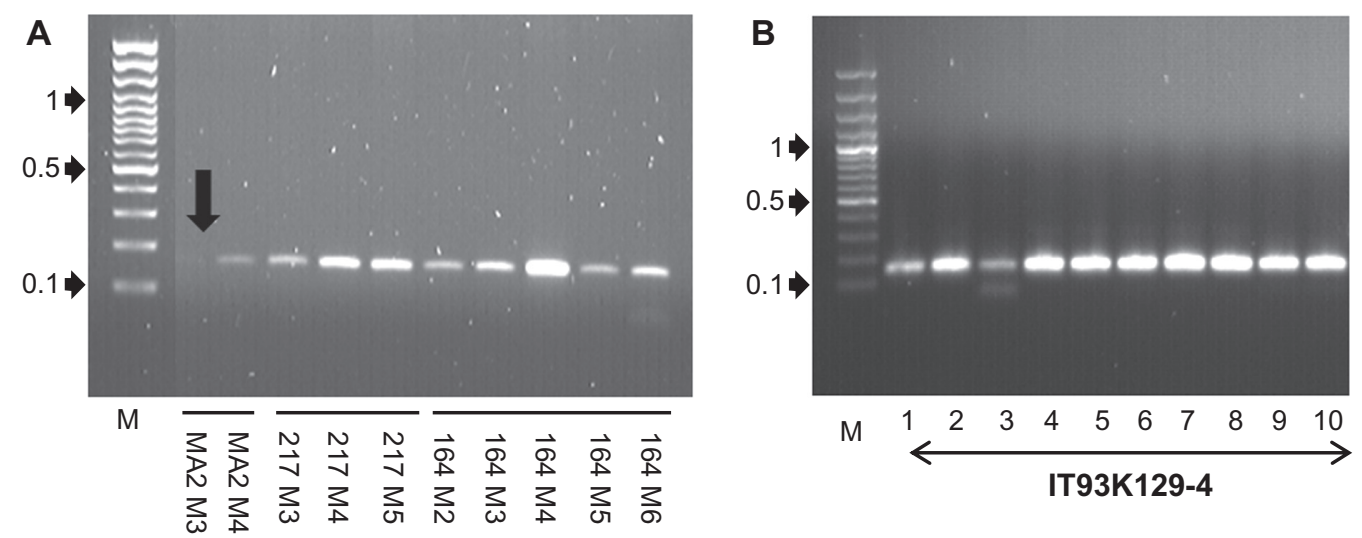

Figure 2 Cowpea DNA used as template in a PCR reaction with primers E5L8 and E5R8 designed to bind within subtraction product E5/8. (A) Genomic DNA from mutant cowpea lines MAI, 2I7, and I64; (B) lanes I to 10 represent DNA of 10 individual control (IT93K I29-4) cowpea plants as template; lane M represents a molecular DNA marker in kbp. Black arrow indicates the absence of a PCR product.

Abbreviation: PCR, polymerase chain reaction.

that all the $100 \mathrm{M} 1$ individuals had a change in at least one copy of this region following irradiation. For the E5/8out fragment, $18 \%$ of the $\mathrm{M} 2$ individuals, from selfed M1 individuals, in which the band was amplified, did not amplify the band. To arrive at this observation, the group of M1 individuals in which the band was amplified needed to comprise $72 \%$ that were heterozygous for a mutation and $28 \%$ that did not have a mutation in this region. Therefore, the region defined by the
E5/8out primers was mutated in 74.5\% (65.5\% heterozygous and $9 \%$ homozygous) of the irradiated plants. Since all of the M1 plants appeared to be heterozygous with the E5/8 primers, no repair could occur. However, $63 \%$ of the individuals in which no band was observed in the M1 had a band in the M2. Therefore, the repair process appears to be much more efficient in the homozygous mutated individuals than in the heterozygous individuals.
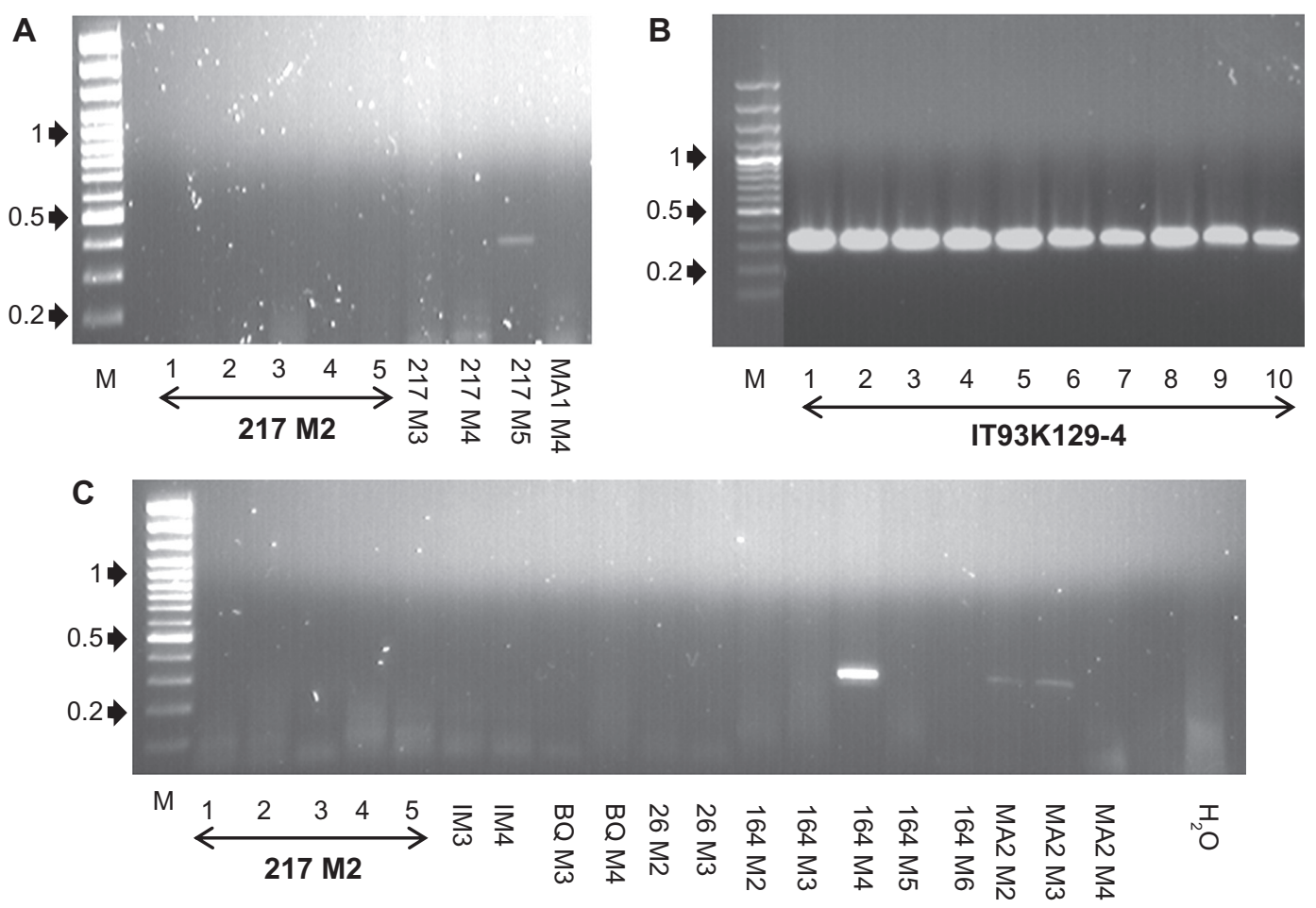

Figure 3 Cowpea DNA used as template in a PCR reaction with primers E5L8out and E5R8 out. (A) Genomic DNA from mutant cowpea lines 2 I 7 (M2-M5) and MAI (M4) used as template. (B) Lanes I to 10 represent DNA of 10 individual control (IT93K I29-4) cowpea plants as template; (C) Genomic DNA from individuals from mutant lines 217, I, BQ, 26, 164, MA2. Lane M represents a molecular DNA marker in kbp.

Abbreviation: PCR, polymerase chain reaction. 


\section{RAPD analysis}

Five of the RAPD primers tested (OPB07, OPB11, OPC11, OPK04, and OPAC19) showed consistent banding patterns in the nonmutated control line, and primers were selected for analysis of the mutated lines. Polymorphisms were detected between irradiated mutants and control individuals (Table 5 and Figure 4). For example, the control nonirradiated RAPD profile for primer OPB11 detected six bands, including SecM, but additional bands are seen in some of the mutant lines (Figure 4). RAPD amplification data were used to obtain a similarity matrix reflecting the genetic relationship between the Vigna mutants and the nonirradiated control line. The dendrogram resulting from the similarity coefficient among the Vigna lines was calculated according to Nei and Li's coefficient ${ }^{29}$ (Figure 5). Similarity levels differed between mutant lines as well as between parent control and mutant lines. It was observed that mutant lines MA2 and 26 differed markedly from the parental control line, which indicates a higher mutation rate in these regions for these two lines.

Several RAPD fragments were excised, isolated, cloned, and sequenced. One of these regions, SecM, was isolated from a nonirradiated control individual, as a representative of a seemingly nonpolymorphic region (Figure 4). SecM together with a second fragment, SecI, showed significant similarity to Medicago truncatula (AC146568) and L. japonicus (AP010349.1) genomic DNAs, respectively, as well as similarity found in the cowpea gene space sequence knowledge database (SecI: Accession No gnl/vuna/35044500; putative cullin and SecM: gnl/ vuna/35034185; aspartic peptidase). Four primers were designed for each of these two fragments. The four primers included two binding on each end and two inside the isolated fragment. One hundred irradiated cowpea M1 individuals were screened with four primer combinations each for primer sets SecI and SecM. The primer combinations included two end primers, two inside primers, one $3^{\prime}$ end and one $5^{\prime}$ inside primer and one $5^{\prime}$ end and one $3^{\prime}$ inside primer. The absence of a PCR amplification product was expected if the parent was homozygous for absence of the target PCR fragment. The presence of a PCR amplification product was expected if the parent was either homozygous or heterozygous for the presence of the PCR target. All the regions using the four sets of primers for SecI and SecM could be amplified from all control individuals. In the M1 analysis, $27 \%$ and $8 \%$ of individuals failed to amplify the internal SecI and SecM regions, respectively.

M1 individuals, showing no amplification of the SecI region, were selfed, M2 seeds were planted, and genomic
Table 5 Amplification products obtained using RAPD technique with DNA from control and mutant cowpea lines

\begin{tabular}{lllll}
\hline Primer & Sequence & $\begin{array}{l}\text { Total of } \\
\text { bands }\end{array}$ & $\begin{array}{l}\text { Polymorphic } \\
\text { bands }\end{array}$ & $\begin{array}{l}\text { Polymorphic } \\
\text { percentage }\end{array}$ \\
\hline OPBII & GTAGACCCGT & 7 & 6 & 86 \\
OPB07 & GGTGACGCAG & 8 & 7 & 88 \\
OPACI9 & AGTCCGCCTG & 6 & 4 & 67 \\
OPK4 & CCGCCCAAAC & 10 & 10 & 100 \\
OPCII & AAAGCTGCGG & 7 & 3 & 43 \\
\hline
\end{tabular}

Notes: PCR were repeated for 10 individuals of each mutant or control line for each of the five primers.

Abbreviations: RAPD, random amplified polymorphic DNAs; PCR, polymerase chain reactions.

DNA was extracted for further PCR screening. Of the resulting M2 individuals screened, 23\% still showed no amplification of the SecI PCR fragment but the fragment was amplified from $77 \%$ of the individuals. Screening of SecM still showed no amplification in $20 \%$ of individuals but the fragment could be amplified in $80 \%$ of M2 individuals. Neither of these values is significantly different from a 3:1 segregation. Therefore, it appears that, in every case, one of the two copies of the region is repaired in every premeiotic cell, giving a segregation ratio equivalent to that expected for the selfing of a heterozygote. However, as noted above, because no band was amplified in the DNA isolated from the parental M1 plants, this repair needed to occur after the isolation of the DNA. The most likely timing of the repair would be during preparation for entry into the reproductive phase, but this hypothesis needs to be directly tested.
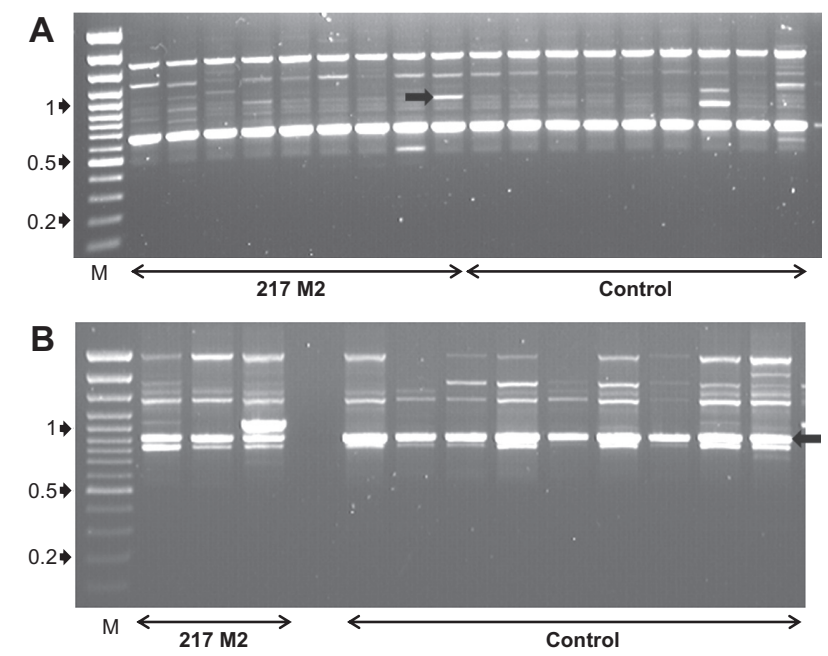

Figure 4 RAPD profile of genomic DNA from individuals from mutant line 217 and control line IT93K I29-4 generated with primer OPBII (A) and OPB07 (B). Lane M represents a molecular DNA marker in kbp. Arrow in $(\mathbf{A})$ indicates position of Secl fragment and arrow in (B) indicates position of SecM fragment. Abbreviation: RAPD, random amplified polymorphic DNAs. 


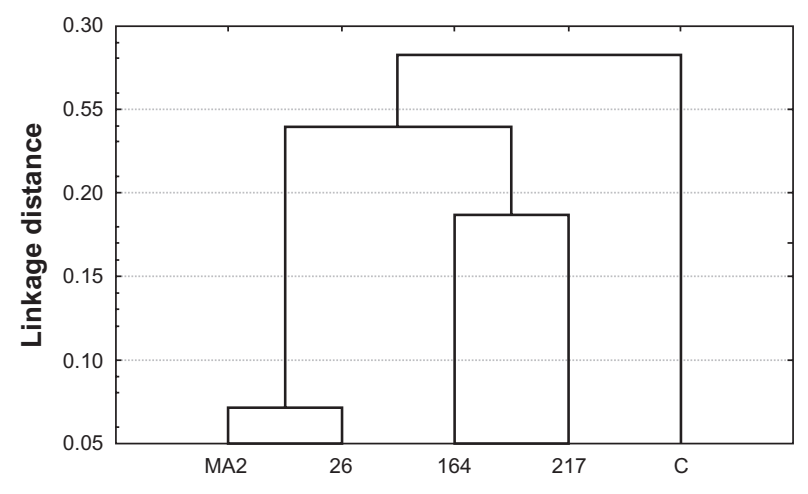

Figure 5 Dendrogram derived from similarity indexes, developed from RADP data, in the mutant lines (MA2, 26, I64, 217) and parental control variety IT93K I 24-9. Abbreviation: RAPD, random amplified polymorphic DNAs.

\section{SSR analysis}

All 10 primer pairs used for the analysis amplified SSR fragments from genomic DNA of control cowpea seeds or plants (data not shown). Analysis of 100 M1 cowpea individuals with the different SSR primer pairs showed no variation in the fragment lengths amplified with each primer pair across the different samples. This SSR-based control was performed to eliminate the possibility that outcrossing was the reason for the variation in mutant genotypes seen in the M1 and M2 generations.

\section{Discussion}

In this study, the RDA technology was successfully used to isolate differences in genomic DNA of radiated cowpea plants. A considerable number ( $40 \%)$ of these isolated fragments had high similarity to chloroplast DNA with nonchloroplast genome sequences at the ends of some of these fragments. Because such mutations would have been lethal to the chloroplast, disrupting gene function and impairing plastid function, we assume that these fragments represent plastid DNA insertions into the nuclear cowpea genome as susceptible targets for irradiation-induced mutations.

Nuclear chloroplast sequences might act as a mutation buffer under various forms of stress. These sequences might firstly be particularly labile because of their position in the genome such as being very receptive regions of the genome. Secondly, they might be recognized as dispensable fragments in some way (such as a specific chromatin structure) so that they provide a sink for mutations and protect more essential regions of the genome.

Our study has provided several lines of evidence for this assumption. RDA subtraction product E2/D had a changed methylation pattern lacking the $M s p$ I restriction site.
Because chloroplast DNA is nonmethylated, ${ }^{31}$ any detected methylated sequences are very likely to represent nuclear copies of plastid DNA inserts. Data from pine trees exposed to ionizing radiation have indicated that such hypermethylation is a possible defense strategy of the plants to survive extreme environments. ${ }^{32}$ Also, the plant genome response to environmental and genetic stresses generates both novel genetic and epigenetic methylation polymorphisms. ${ }^{33}$

Further, one fragment (E5/8) derived from a pooled DNA sample of two mutant lines (MA1 and 217) shared sequence similarity with the chloroplast genome but with a 12 bp 3' flanking region which is not part of the chloroplast DNA. E5/8 showed a high mutation rate $(100 \%)$ and a relatively low "repair" rate $(62.5 \%)$ in the M1 and M2 generation individuals, respectively. Actively transcribed genes are more rapidly repaired than nonexpressed genome fragments to maintain DNA integrity. ${ }^{34}$ Therefore, the lower repair rate of the E5/8 very likely indicates that this DNA is not part of a transcribed nuclear gene.

We also investigated "repair" of mutated DNA. "Repair" is used in the context of this communication to identify events where the mutation, associated with the irradiation treatment, disappears in subsequent generations. One of the sequences identified by RDA could be possibly a retrotransposon (AAV88076.1). Lack of amplification of this sequence might be due to the insertion of a transposable element which might have resulted in a fragment too long to be amplified under the amplification conditions used here. Any "repair" event that included the excision of the inserted transposon would return the amplified fragment to approximately the previous size with the addition of any duplicated regions from the insertion site that is a remaining footprint. Such a footprint would not be large enough to make a difference to the size of the amplified product but could be tested by sequencing the "repaired" amplified band to determine the difference, if any, from the progenitor fragment. Retrotransposons are known to be activated when plant genomes experience stress. ${ }^{35,36}$ However, future characterization of the "repaired" sites might allow a more specific insight into the "repair" process and if retrotransposons are involved.

By using RAPD technology, we could further demonstrate that "repair" occurs in following generations of radiation-induced mutants. Polymorphisms were detected between irradiated mutants and control individuals when RAPD primers were used to detect mutations by 
appearance/disappearance of amplified fragments. In particular, MA2 and 26 differed markedly from the parental control line, with altered RAPD fragment amplification in these lines indicating the occurrence of mutations. The SecM fragment arose at a relatively low rate in the M1 generation (8\% not amplifying the band). This is not unexpected because both copies of this region need to be mutated to lose the amplification product. If the events are independent, then this translates to a mutation rate at this locus of 0.283 . This value is similar to that observed for the region identified by $\mathrm{E} 5 / 8$ which had a mutation rate to homozygosity of 0.32 . The "repair" rate in the M2 generation individuals was high $(80 \%)$. One possibility is that amplification from heterozygous plants was insufficient to detect the band. However, if this was the underlying cause, then the expected "repair" rate would be $25 \%$ (only the homozygotes comprising $25 \%$ of the selfed progeny) could be detected but the observed rate was $77 \%$. In contrast, the SecI fragment had a higher mutation rate in the M1 generation $(27 \%$ not amplifying the band, which is equivalent to a mutation rate of 0.52 ) but was also "repaired" at similar relatively high "repair" rate in the M2 individuals (77\%). Again this is not consistent with the mutation following irradiation resulting in heterozygous plants in which the band could not be detected. Therefore, the two regions are mutated by irradiation at different rates, but the proportions that are "repaired" are equivalent. Thus the genomic regions that are differentially targeted for mutation are not similarly differentiated by the "repair" process. In the characterization of sweet potato radiation-induced mutations, RAPDs were used to develop a phylogeny of the mutants. ${ }^{22}$ Although no details of the distribution of polymorphic bands is given, because there is a grouping of the mutants that separate them from the irradiated parent line, the members of each group need to have some shared RAPD polymorphisms. This is consistent with the observations reported here that the rate of mutation across the genome is not uniform. One question that could be addressed using these mutants is, are any of the RAPD polymorphisms nuclear localized chloroplast sequences? If an affirmative answer is obtained, then it would add weight to the proposal that this compartment of the genome does have some special sensitivity to ionizing radiation.

We used the SSR technology to eliminate any outcrossing as a source of "repair" events. SSRs are known to display variation between different genomes due to mutations occurring of slippage of the DNA polymerase enzyme during DNA replication. However, these types of events do not appear to be frequent during radiation treatment. ${ }^{37}$ Analysis of 10 different SSR loci in 100 different mutant cowpea M1 individuals originating from the same parent also showed in our study no detectable variation in fragment size in our analyzed lines. Results reported here are therefore consistent with previous findings ${ }^{37}$ in rice where induced mutants had SSR profiles identical to their parents. Any possible detectable variations due to outcrossing, which results in SSR polymorphisms, could then have been interpreted as equivalent to the results found for salt-tolerant sorghum, where SSRs detected genetic changes induced by gamma rays ${ }^{38}$ but subsequently interpreted as resulting from outcrossing.

We also considered it as important to include nontransmissible mutations in our study when analyzing the effect of irradiation on the plant genome. Previous large-scale DNA screening revealed that only a few of several hundred mutations were transmitted to the progeny. ${ }^{39}$ Therefore, we included a second set of irradiated M1 cowpea seeds in our analysis. Of the $100 \mathrm{M} 1$ individuals used for mutation screening, 82 germinated and initially grew normally but only 44 produced seeds, and the other 38 plants died before producing seeds. M1 (9\%) and M2 plants (13\%) of the screened individuals only showed limited germination and growing abilities, and a small number of the M1 seeds (7\%) did not germinate at all. This result is consistent with the known effects of gamma rays on the growth of M1 plants, which include reduction of fertility, seed set, plant height, and abnormal growth forms, such as chlorosis and curly leaves, with a correlation between dose and altered phenotype..$^{37,40}$

In summary, our study has shown that in cowpea a large proportion of induced nonlethal mutations might occur within nuclear plastid insertions and a relatively high repair rate occurs in mutated plants in the progeny. The high frequency of alterations in this compartment of the nuclear genome is consistent with the suggestion that the regions in which chloroplast sequences integrate into the genome are particularly labile. ${ }^{41}$ These nuclear-located chloroplast sequences and their modification might be analogous to the movement of transposable elements, as has also previously been reported when analyzing tobacco plants generated in vitro. ${ }^{42}$ However, these labile regions may not be randomly distributed throughout the genome, leading to unequal distribution of mutations in the plant genome in response to irradiation. 


\section{Acknowledgments}

The work was funded by the International Atomic Energy Agency (Austria). Irradiation dosage determination and mutant collection were done by Spreeth et al at the Agricultural Research Council, South Africa, as part of a project funded by the International Atomic Energy Agency. We thank the Agricultural Research Council for providing us with the control and mutant cowpea seeds. We thank Farida Samodien at the Institute for Plant Biotechnology, Stellenbosch University, for molecular data collection done during the PCR screening of samples.

\section{Disclosure}

The authors report no conflicts of interest in this work.

\section{References}

1. Fery FL. New opportunities in Vigna. In: Janick J, Whipkey A, editors. Trends in New Crops and New Uses. Alexandria, VA: ASHS Press; 2002.

2. Ikushima T. Somatic mutation in cereals. Mutat Res/Fund Mol Mech Mutagenesis. 1987;181:199-207.

3. Xiaoming S, Ke L, Yanli N, Genfa Z. Biochemistry and genetic analysis of bioeffects of low energy $\mathrm{N}+$ implantation and $\gamma$-radiation on Arabidopsis thaliana. Front Biol China. 2006;1:41-45.

4. Ahloowalia BS, Maluszynski M. Induced mutations - a new paradigm in plant breeding. Euphytica. 2001;118:167-173.

5. Bruggemann E, Handwerger K, Essex C, Storz G. Analysis of fast neutron-generated mutants at the Arabidopsis thaliana HY4 locus. Plant J. 1996;10:755-760.

6. Shirley BW, Hanley S, Goodman HM. Effects of ionizing radiation on a plant genome: Analysis of two Arabidopsis thaliana testa mutations. Plant Cell. 1992;4:333-347.

7. Terato H, Tanaka R, Nakaarai Y, et al. Quantitative analysis of isolated and clustered DNA damage induced by gamma-rays, carbon ion beam, and iron ion beams. J Radiat Res. 2008;49:133-146.

8. Tuteja N, Singh MB, Misra MK, Bhalla PL, Tuteja R. Molecular mechanisms of DNA damage and "repair": Progress in plants. Crit Rev Biochem Mol Biol. 2001;36:337-397.

9. Koornneef M, Dellaert LWM, van der Veen JH. EMS- and radiationinduced mutation frequencies at individual loci in Arabidopsis thaliana (L.), Heynh. Mutat Res. 1982;93:109-123.

10. Roy S, Begum Y, Chakraborty A, Raychaudhuri S. Radiationinduced phenotypic alterations in relation to isozymes and RAPD markers in Vigna radiate (L.) Wilczek. Int J Radiat Biol. 2006;82: 823-832.

11. Genfa Z, Ke L, Xiaoming S, Yanli N, Jun Z, Hongyu Z, Ting L. Comparative study on effects of low energy $\mathrm{N}$ implantation and $\gamma$-ray radiation on heredity and development of Arabidopsis thaliana. Plasma Sci Tech. 2005;7:2879-2884.

12. Rogozin IB, Pavlov YI. Theoretical analysis of mutation hotspots and their DNA sequence context specificity. Mutat Res. 2003;544:65-85.

13. Cecchini E, Mulligan BJ, Covey SN, Milner JJ. Characterization of gamma irradiation-induced deletion mutations at a selectable locus in Arabidopsis. Mutat Res. 1998;401:199-206.

14. Hada M, Georgakilas AG. Formation of clustered DNA damage after high-LET irradiation: A review. J Radiat Res. 2008;49:203-210.

15. Terato H, Ide H. Clustered DNA damage induced by heavy ion particles. Biol Sci Space. 2004;18:206-215.

16. MacLeod MR. Analysis of an allelic series of mutant at the $r$ locus of pea. PhD Thesis, University East Anglia, UK; 1994.
17. Bhattacharyya MK, Smith AM, Ellis THN, Hedley C, Martin C. The wrinkled-seed character of peas described by Mendel is caused by a transposon-like insertion in a gene encoding starch branching enzyme. Cell. 1990;60:115-122.

18. Molle G. The influence of seed treatment with gamma radiation on plant yield. Int J Appl Radiat Isot. 1965;16:397-404.

19. Lisitsyn N, Lisitsyn N, Wigler M. Cloning the differences between two complex genomes. Science. 1993;259:946-950.

20. Ushijima U, Morimuta K, Osoya Y, et al. Establishment of methylationsensitive-representational difference analysis and isolation of hypo- and hypermethylated genomic fragments in mouse liver tumors. Proc Natl Acad Sci U S A. 1997;94:2284-2289.

21. Michiels L, Van Leuven F, Van den Oord JJ, De Wolf-Peeters C, Delabie J. Representational difference analysis using minute quantities of DNA. Nucleic Acid Res. 1998;26:3608-3610.

22. Shin JM, Kim BK, Seo SG, et al. Mutation breeding of sweet potato by gamma-ray radiation. Afri J Agric Res. 2011:6:1447-1454.

23. Atak C, Alikamanoglu S, Acik L, Canbolat Y. Induced plastid mutations in soybean plant (Glycine max L. Merrill) with gamma radiation and determination with RAPD. Mutat Res. 2004;556:35-44.

24. Kuroda S, Yano H, Koga-Ban Y, et al. Identification of DNA polymorphism induced by X-ray and UV irradiation in plant cells. Japan Agri Res Quar. 1999;33:223-226.

25. Vorster JB, Kunert KJ, Cullis CA. Use of representational difference analysis for the characterization of sequence differences between date palm varieties. Plant Cell Rep. 2002;21:271-275.

26. Oh TJ, Cullis CA. Labile DNA sequences in flax identified by combined sample representational difference analysis (csRDA). Plant Mol Biol. 2003;52:527-536.

27. Zane L, Bargelloni L, Patarnello T. Strategies for microsatellite isolation: A review. Mol Ecol. 2002;11:1-16.

28. Li K, Nie Y, Zhang D, Zhang J, Zhang G. AFLP analysis of Arabidopsis thaliana treated by low-energy ion beam. IEEE Trans Plasma Sci. 2007;35:454-459.

29. Nei M, Li W-H. Mathematical model for studying genetic variation in terms of restriction endonucleases. Proc Natl Acad Sci U S A. 1979;76:5269-5273.

30. Ahlert D, Stegemann S, Kahlau S, Stephanie Ruf S, Bock R. Insensitivity of chloroplast gene expression to DNA methylation. Mol Genet Genomics. 2009;282:17-24.

31. Fojtova M, Kovarik A, Matyasek R. Cytosine methylation of plastid genome in higher plants. Fact or artefact? Plant Sci. 2001;160:585-593.

32. Kovalchuk O, Burke P, Arkhipov A, et al. Genome hypermethylation in Pinus sylvestris of Chernobyl - a mechanism for radiation adaptation? Mutat Res. 2003;529:13-20.

33. Lukerns LN, Zhan S. The plant genome's methylation status and response to stress: Implications for plant improvement. Curr Opin Plant Biol. 2007; 10:317-322.

34. Bohr VA, Smith CA, Okumoto DS, Hanawalt PC. DNA repair in an active gene: Removal of pyrimidine dimers from the DHFR gene of $\mathrm{CHO}$ cells is much more efficient than in the genome overall. Cell. 1985;40:359-369.

35. Grandbastien MA. Activation of plant retrotransposons under stress conditions. Trends Plant Sci. 1998;3:181-187.

36. Pouteau S, Huttner E, Grandbastien MA, Caboche M. Specific expression of the tobacco Tnt1 retrotransposon in protoplasts. EMBO J. 1991;10:1911-1918.

37. Fu HW, Li YF, Shu QY. A revisit of mutation induction by gamma rays in rice (Oryza sative L.): Implications of microsatellite markers for quality control. Mol Breed. 2008;22:281-288.

38. Tabosa JN, Colaco W, Reis OV, Simplicio JB, Dias FM. Sorghum genotypes tolerant to soil salinity - progenies developed under gamma ray doses. J Semi-Arid Trop Agric Res. 2007;5:1-5.

39. Naito K, Kusaba M, Shikazono N, et al. Transmissible and nontransmissible mutations induced by irradiating Arabidopsis thaliana pollen with $\gamma$-rays and carbon ions. Genetics. 2005;169:881-889. 
40. Abdel-Hady MS, Okasha EM, Soliman SSA, Talaat M. Effect of gamma radiation and gibberellic acid on germination and alkaloid production in Atropa belladonna L. Aust J Basic Appl Sci. 2008;2:401-405.

41. Cullis CA, Vorster BJ, Van Der Vyver C, Kunert KJ. Transfer of genetic material between the chloroplast and nucleus: How is it related to stress in plants? Ann Bot. 2009;103:625-633.
42. Stegemann S, Hartmann S, Ruf S, Bock R. High-frequency gene transfer from the chloroplast genome to the nucleus. Proc Natl Acad Sci U SA. 2003;100:8828-8833.

Research and Reports in Biology

\section{Publish your work in this journal}

Research and Reports in Biology is an international, peer-reviewed, open access journal publishing original research, reports, editorials, reviews and commentaries on all areas of biology including animal biology, biochemical biology, cell biology, ecological studies, evolutionary biology, molecular biology, plant science and botany. The

\section{Dovepress}

manuscript management system is completely online and includes a very quick and fair peer-review system. Visit http://www.dovepress $\mathrm{com} /$ testimonials.php to read real quotes from published authors. 